Aim of the study: The present study aims to explore the value of application of hepaticojejunostomy in surgical treatment of Bismuth-Corlette type III hepatic hilar cholangiocarcinoma.

Material and methods: The clinical data of hepaticojejunostomy in 6 patients with Bismuth-Corlette type III hepatic hilar cholangiocarcinoma from January 2008 to October 2011 were retrospectively analyzed. There were 5 males and 1 female, aged 45-69 years, on average 58 years old. There were 3 cases of Bismuth-Corlette type IIla and 3 cases of Bismuth-Corlette type IIIb.

Results: The 6 patients all successfully underwent hepaticojejunostomy, with operation time of 160-310 min, on average $210 \pm 28 \mathrm{~min}$. One patient suffered a small amount of bile leakage $(3-20 \mathrm{ml})$ and his drainage tube was taken out after being cured with conservative treatment for 18 days. Three patients showed postoperative mild increases of aspartate aminotransaminase (AST), alanine transaminase (ALT) and AKP, which were improved after drug treatments. There was no biliary stricture in the 6 patients after follow-up for 3-18 months.

Conclusions: Hepaticojejunostomy was simple and flexible, with a good effect on biliary reconstruction of Bismuth-Corlette type III hepatic hilar cholangiocarcinoma. It solved the difficulties in multiple choledochojejunostomy, and with less complications; therefore, it is worth being popularized.

Key words: hepaticojejunostomy, bile duct neoplasms, stricture.

\section{Treatment of hilar cholangiocarcinoma of Bismuth-Corlette type III with hepaticojejunostomy}

\author{
Bao-Chun Lu, Pei-Tu Ren
}

Department of Hepatobiliary Surgery, Shaoxing People’s Hospital, Zhejiang, China

\section{Introduction}

With the improvement of modern imaging and surgical techniques and progress in perioperative management, early diagnosis and surgical treatment of hilar cholangiocarcinoma have improved significantly. Relevant data show that about $50 \%$ of clinical cases were Bismuth-Corlette type III [1]. Nearly all surgeons in Europe and the United States and Japan and other countries advocate using major hepatectomy (such as hemihepatectomy or trisegmentectomy) for treatment of this type of liver hilar cholangiocarcinoma, but the operative mortality of major hepatectomy is up to about 10\% [2-12]. In particular, for patients with preoperative jaundice, the mortality is up to $17-23 \%$, the incidence of postoperative complications is up to $40-71 \%$, and the 5 -year survival rate is only $20-30 \%$ [1-12]. Even liver transplantation cannot improve the longterm survival of patients [13-15]. The tertiary hepatic duct and its distal hepatic duct and liver parenchyma in patients with Bismuth-Corlette III hilar cholangiocarcinoma showed no tumour infiltration; thus it is unnecessary to remove it all at once on a large scale. Clinicians generally use local small-scale liver resection surgery instead of hemihepatectomy, but 3-9 bile duct openings will be left in the hepatic segment surface after the surgery. Commonly used in clinical practice is carrying out Roux-en-Y anastomosis with the jejunum after orthomorphia of various hepatic ducts or carrying out multiport anastomosis for each hepatic duct with the jejunum [16]. The anastomosis technique is not only complicated to perform but also time-consuming, and anastomotic stricture, biliary fistula [17, 18] and reflux cholangitis [19] are easy to occur postoperatively. After years of clinical research, we have designed a simple enterohepatic consistent, in which only the posterior wall of the bile duct is sutured, the anterior wall is sutured with the liver tissue, and there is no need to place a bile duct support drainage tube, and it is more simple and convenient for surgical procedures of smaller multiple bile ducts. From January 2008 to October 2011, a total of six cases of Bismuth-Corlette III hepatic hilar cholangiocarcinoma underwent enterohepatic anastomosis in our hospital. No anastomotic stenosis occurred after the surgery, the results were satisfactory, and now our experiences are described below.

\section{Materials and methods}

\section{General data}

There were six patients in this group: 5 males and 1 female, aged from 45 to 69 years, on average 58 years old. Three cases were Bismuth-Corlette IIIa, and 3 cases were Bismuth-Corlette IIIb (Table 1). Preoperative examinations included routine liver, kidney function and other routine examinations, ultrasound, CT and magnetic resonance for biliary imaging (Fig. 1). This study was conducted in accordance with the Declaration of Helsinki and with approval from the Ethics Committee of Shaoxing People's Hospital. Written informed consent was obtained from all participants. 
Table 1. General data of the patients who underwent surgical resection

\begin{tabular}{|c|c|c|c|c|c|c|}
\hline Case & 1 & 2 & 3 & 4 & 5 & 6 \\
\hline Gender & Male & Male & Female & Male & Male & Male \\
\hline Age & 45 & 55 & 69 & 52 & 61 & 66 \\
\hline Type (Bismuth-Corlette) & IIla & $\| I I b$ & IIla & IIIa & IIIb & IIIb \\
\hline Preoperative bilirubin (mmol/l) & 260 & 296 & 180 & 266 & 276 & 248 \\
\hline Intraoperative blood loss (ml) & 180 & 100 & 900 & 300 & 200 & 300 \\
\hline Operation time & 180 & 160 & 310 & 220 & 190 & 200 \\
\hline Post-operative bile leakage & No & No & Yes & No & No & No \\
\hline $\begin{array}{l}\text { Pathological diagnosis } \\
\text { (Extent of differentiated adenocarcinoma) }\end{array}$ & well & moderate & poor & poor & moderate & moderate \\
\hline
\end{tabular}

\section{Surgery}

There were 5 patients in this group with total bilirubin greater than $200 \mathrm{mmol} / \mathrm{l}$, with duration of greater than 4 weeks; 2 patients received PTCD first before the surgery, 3 patients received nasobiliary drainage first, and then received surgical treatment until the total bilirubin declined to a normal level.

\section{Hepatectomy range}

Three patients received segment IV hepatectomy, and the other 3 patients received segment IV + segment $V$ hepatectomy; patients with the most hepatic duct openings of the hepatic segment surface were those who received segment IV + segment $\mathrm{V}$ hepatectomy, 3-5 openings on the right hepatic segment surface, and 2-5 on the left hepatic segment surface; duct openings in the hepatic surgical wound close to each other may be corrected by suturing, to form a large bile duct opening, while the hepatic duct openings far away from each other were not corrected, and only the stumps were pruned.

\section{Biliary tract reconstruction}

For patients who received segment IV or segment IV + segment $\mathrm{V}$ hepatectomy, the conventional biliary reconstruction method is complicated and time-consuming, and there is a high incidence of postoperative complications. In view of this situation, we have adopted a new method of biliary reconstruction, and the surgery was completed by the following steps: 1) treatment of liver sections to completely expose the bile duct stumps, but freeing of the bile duct stumps is not strictly required, to prevent affecting blood supply; 2) pruning the bile duct stumps until the bile duct is pruned to achieve normal blood supply and being smooth; 3) according to Roux-en-Y design, the cutting-off of the distal jejunum will be lifted up to the hepatic segment since the colon end side was closed by suturing, the side is cut open for anastomosis, and the appropriate anastomotic size should be able to fully include the bile duct stump of the hepatic segment. Two corners of the intestinal side and fascia tissue, liver capsule, or portal vein sheath of the hepatic segment are sutured with a stitch of Prolene with five 0 and a stitch of Prolene with three $0 ; 4$ ) anastomosis started with the posterior wall, on one side, 5 pieces of 0 Prolene are used for continuous full layer suturing from the liver tissue and portal vein sheath $1 \mathrm{~cm}$ below the bile duct stump with the bowel wall; if the suture comes to the underneath of the bile duct posterior wall, the posterior wall of the bile duct can be sutured together, the suture force should be moderate, being careful not to cut the Prolene, and when the posterior wall has been sutured, the Prolene on this side should be knotted with Prolene on the other side; 5 ) if the diameter of the bile duct stump is greater than $2 \mathrm{~mm}$, a silicone tube should not be placed, and a silicone tube was placed only in one case in this group; 6) another Prolene with three 0 is used for continuous suture of the full thickness of the jejunum anterior wall with liver tissue of the hepatic segment, on the one hand, to ensure that the bile duct stump is not pulled, while on the other hand, more liver tissue should be sutured, to include the liver capsule as far as possible; 7) jejunum end anastomosis is conducted at $50 \mathrm{~cm}$ below the anastomotic openings; 8) an abdominal drainage tube is placed under the enterohepatic anastomotic openings. The patients should be treated like those undergoing conventional abdominal surgery; broad spectrum antibiotics should be applied to prevent infection, and the abdominal drainage tube should be removed after gastrointestinal decompression to anus exhaust when there is no liquid drainage. 


\section{Results}

\section{General data}

As shown in Table 1, the operations for the 6 patients were successful. The operation time was 160-310 min, with an average operation time of $210 \pm 28 \mathrm{~min}$. Each patient was placed with an abdominal drainage tube, and the removal time was 5-18 days after the surgery, with an average time of $7 \pm 1.2$ days. Intraoperative blood loss was $100-900 \mathrm{ml}$, and the 6 patients showed no bile duct stenosis during the 3 to 18 months of follow-up.

\section{Postoperative complications}

There was no wound infection, intra-abdominal infection or pulmonary infection. One patient showed a small amount of bile leakage; the daily maximum amount was $20 \mathrm{ml}$, it spontaneously decreased 12 days later, and the drainage tube was removed 18 days later. Three patients showed a mild degree of AST, ALT and AKP increase, and they were improved after drug treatment.

\section{Pathological diagnosis}

One patient had well-differentiated adenocarcinoma; 3 patients had moderately differentiated adenocarcinoma; 2 patients had poorly differentiated adenocarcinoma; 1 patient showed a positive surgical margin after bile duct surgery, while the other 5 were negative.

\section{Discussion}

The intrahepatic invasion range of tumor in the BismuthCorlette III hilar cholangiocarcinoma normally includes the primary or secondary hepatic duct; in order to achieve the purpose of complete resection of the tumor and trying to save the residual liver function, hepatic segments of $\mathrm{IVb}+\mathrm{IV}+\mathrm{V}$ or IV $+\delta+I$ are normally needed to be removed in surgery [2]. The problems directly deriving from this are not only that 2 to 3 hepatic segment surfaces are left, but also 3-9 bile duct openings are left; thus it is very difficult to carry out surgical repair [20]. In this case, the clinician will usually use two methods: 1) if the hepatic duct stump openings are close to each other, Roux-en-Y anastomosis will be conducted for the corrected hepatic duct and the jejunum during the surgery [4], as the openings of the secondary hepatic duct are fine, correction is difficult; 2 ) if the hepatic duct stump openings are distant from each other, each hepatic duct opening will be anastomosed with the intestinal canal for multi-port anastomosis to reconstruct the bile duct, but the method is complicated and difficult to implement. No matter what method is used, anastomotic stricture is easy to occur after surgery, and once anastomotic stricture occurs, it will be more difficult to handle; the consequences must be recurrent cholangitis and biliary cirrhosis and even liver failure and death. In view of this, we have adopted a hepatoenterostomy in which the bile duct is directly exposed to the enteric cavity instead of anastomosis; as the bile duct is anastomosed in this surgery, there is no need to carry out bile duct orthopedic surgery, there is suture stimulation to the bile duct after pruning, it is less prone to have anastomotic stricture, and the surgery is simple, easy, and can significantly shorten the surgical time. The surgical method was adopted in the six cases in this group, and no anastomotic stricture occurred in the 3 to 18 months of follow-up.

Anastomotic stricture is avoided with the application of enterohepatic anastomosis, but due to poor tensile strength of liver tissue, how to prevent the occurrence of anastomotic leakage is particularly important. Of the six cases in this group, only one case showed a small amount of biliary fistula and healed spontaneously after short-term drainage.

Through the experience of six cases, we believe that the following points should be noted during surgical procedures: 1) the intestinal suture should be sutured and tissues fixed with great anti-tensile strength in the hepatic segment as far as possible, such as the sheath-like tissue in the hepatic segment and liver capsule tissue; if not, the intestinal canal can be directly sutured to the vascular sheath; 2) the anterior and posterior wall of the anastomotic openings should be continuously sutured and Prolene sutures should be used, as they are easy to tighten and do not easily cause liver tissue cuts; for the suture of the anterior wall, liver tissue should be sutured as much as possible, and the liver capsule should be sutured together; 3) anastomotic water-filling test: when anastomosis is completed, the distal bowel end of the anastomotic opening should be clipped, and a needle should be used to inject an appropriate amount of saline to the proximal bowel, and check whether there is infiltration of fistula at the anastomotic openings; 4) application of hepatic duct stent: for those with fine hepatic duct openings (less than $2 \mathrm{~mm}$ ) or with incomplete liver capsule and sheath tissue, a hepatic duct stent thereof should be placed to prevent the occurrence of anastomotic leakage; 5) the placement of a peritoneal drainage tube is very important for the prevention of anastomotic leakage: the placement of a drainage tube can not only diagnose anastomotic leakage, but also can ensure smooth drainage, to prevent the emergence of peritonitis, and thus to avoid reoperation.

As the hepatic duct and the intestinal mucosa do not need to be anastomosed in enterohepatic anastomosis, and thus it is not limited by hepatic duct thickness and stump length, in theory, it is suitable for any patient with hepatic duct jejunostomy. However, it is of little advantage in terms of a level 1 hepatic duct and jejunum anastomosis and it is generally not used in these situations, but it will show its superiority for patients with a number of hepatic duct stumps of which the inner diameter is smaller and distance is long.

In short, the enterohepatic anastomotic method is simple and flexible, is especially suitable for biliary reconstruction in patients with Bismuth-Corlette type III hilar cholangiocarcinoma, fewer complications have been observed, and it is of important clinical value.

The authors declare no conflict of interest.

\section{References}

1. Kondo S, Hirano S, Ambo Y, Tanaka E, Okushiba S, Morikawa T, Katoh $H$. Forty consecutive resections of hilar cholangiocarcinoma with no postoperative mortality and no positive ductal margins. Ann Surg 2004; 240: 95-101. 
2. Witzigmann H, Berr F, Ringel U, et al. Surgical and palliative management and outcome in 184 patients with hilarcholangiocarcinoma. Ann Surg 2006; 244: 230-9.

3. Belghiti J, Ogata S. Preoperative optimization of the live for resection in patients with hilar cholangiocarcinoma. HPB (Oxford) 2005 7: 252-3.

4. Parikh AA, Abdalla EK, Vauthey JN. Operative considerations in resec tion of hilar cholangiocarcinoma. HPB (Oxford) 2005; 7: 254-8.

5. Nishio $H$, Nagino $M$, Nimura Y. Surgical management of hilar cholangiocarcinoma: the Nagoya experience. HPB (Oxford) 2005; 7: 259-62.

6. Yokoyama Y, Nagino M, Nishio H, Ebata T, Igami T, Nimura Y. Recent advances in the treatment of hilar cholangiocarcinoma: portal vein embolization. J Hepatobiliary Pancreat Surg 2007; 14: 447-54.

7. Nagino M, Kamiya J, Arai T, Nishio H, Ebata T, Nimura Y. "Anatomic" right hepatic trisectionectomy (extended right hepatectomy) with caudate lobectomy for hilarcholangiocarcinoma. Ann Surg 2006; 243: 28-32.

8. Hirano S, Tanaka E, Shichinohe T, et al. Treatment strategy for hilar cholangiocarcinoma, with special reference to the limits of ductal resection in right-sided hepatectomies. J Hepatobiliary Pancreat Surg 2007; 14: 429-33.

9. Rea DJ, Munoz-Juarez M, Farnell MB, Donohue JH, Que FG, Crownhart B, Larson D, Nagorney DM. Major hepatic resection for hilar cholangiocarcinoma: analysis of 46 patients. Arch Surg 2004; 139: 514-25.

10. Jonas S, Benckert C, Thelen A, Lopez-Hänninen E, Rösch T, Neuhaus P. Radical surgery for hilar cholangiocarcinoma. Eur J Surg Oncol 2008; 34: 263-71.

11. Otto G. Diagnostic and surgical approaches in hilar cholangiocarcinoma. Int J Colorectal Dis 2007; 22: 101-8.

12. Dinant S, Gerhards MF, Rauws EA, Busch OR, Gouma DJ, van Gulik TM. Improved outcome of resection of hilar cholangiocarcinoma (Klatskin tumor). Ann Surg Oncol 2006; 13: 872-80.

13. Pichlmayr R, Ringe B, Lauchart W, Bechstein WO, Gubernatis G, Wag ner E. Radical resection and liver grafting as the two main components of surgical strategy in the treatment of proximal bile duct cancer. World J Surg 1988; 12: 68-77.

14. Thelen A, Neuhaus P. Liver transplantation for hilar cholangiocarcinoma. J Hepatobiliary Pancreat Surg 2007; 14: 469-75.

15. Robles R, Figueras J, Turrión VS, et al. Spanish experience in liver transplantation for hilar and peripheral cholangiocarcinoma. Ann Surg 2004; 239: 265-71.

16. Leonardi MI, Ataide EC, Boin IF, Leonardi LS. Role of choledochojejunostomy in liver transplantation. Transplant Proc 2005; 37: 1126-8.

17. Moellmann B, Ruhnke M, Kremer B. Cholangio-duodenal interposition of an isolated jejuna segment after central resection. Hepatobiliary Pancreat Dis Int 2004; 3: 259-64.

18. Klaus A, Weiss H, Kreczy A, Eigentler A, Neher C, Margreiter R, Pernthaler $\mathrm{H}$. A new biliodigestive anastomosis technique to prevent reflux and stasis. Am J Surg 2001; 182: 52-7.

19. Jurim O, Shaked A, Rose S, Busuttil R. Volvulus of the Roux-en-Y hepaticojejunostomy. A rare complication manifested by recurrent ascending cholangitis. Int Surg 1994; 79: 176-7.

20. Buell JF, Cronin DC, Funaki B, Koffron A, Yoshida A, Lo A, Leef J, Millis JM. Devastating and fatal complications associated with combined vascular and bile duct injuries during cholecystectomy. Arch Surg 2002; 137: 703-10.

\section{Address for correspondence}

\section{Bao-Chun Lu}

Department of Hepatobiliary Surgery

Shaoxing People's Hospital

No. 568 Zhongxing Road

Shaoxing 312000, Zhejiang, China

tel. 86-575-88229083

e-mail: bcptcn@163.com

Submitted: $\quad 11.08 .2012$

Accepted: $\quad 30.10 .2012$ 